\title{
Central Nervous System Involvement in Patients
} with Diffuse Large B Cell Lymphoma: Analysis of the Risk Factors and Prognosis from a Single-Center Retrospective Cohort Study

This article was published in the following Dove Press journal:

Cancer Management and Research

\section{Jingjing $M a \mathbb{D}^{1, *}$ \\ Qing $\operatorname{Li} \mathbb{D}^{1, *}$ \\ Jie Shao (1D' \\ Yan $\mathrm{Ma}^{2}$ \\ Zhiguang $\operatorname{Lin}^{2}$ \\ Hui Kang' \\ Bobin Chen ${ }^{2}$}

'Department of Hematology, Huashan Hospital North, Fudan University, Shanghai 201907, People's Republic of China; ${ }^{2}$ Department of Hematology, Huashan Hospital, Fudan University, Shanghai 200040, People's Republic of China

*These authors contributed equally to this work
Correspondence: Bobin Chen Department of Hematology, Huashan Hospital, Fudan University, Shanghai 200040, People's Republic of China Tel +86-2I-52889999

Email bbchen@fudan.edu.cn
Purpose: The aim of this study was to identify the risk factors for central nervous system (CNS) involvement in systemic diffuse large B-cell lymphoma (DLBCL) patients and to explore prognostic for DLBCL patients with CNS involvement (relapse or progression).

Patients and methods: This was a retrospective cohort study in our hospital. Data were collected from all DLBCL patients diagnosed in our institutes from January 2013 to June 2018. Clinical information was collected from medical records.

Results: The participants included 138 patients with DLBCL. Among them, 38 patients were diagnosed as CNS lymphoma, including 15 patients exhibited CNS involvement while DLBCL was pathologically confirmed, and 23 patients developed CNS lymphoma during or after initial chemotherapy. The median disease-free interval to CNS involvement was 13 months. Multivariate analysis identified elevated serum lactate dehydrogenase $(\mathrm{LDH})$ level [hazard ratio $(\mathrm{HR})=4.035$; 95\% confidence interval (95\% CI): 1.147-14.195] was an independent predictor of CNS involvement. The median progression-free survival (PFS) and overall survival (OS) time of DLBCL patients with CNS involved were 12.5 months and 22 months, respectively. Multivariate prognostic analysis showed that eastern cooperative oncology group (ECOG) score $>2(\mathrm{P}=0.018$; $\mathrm{HR}=7.333$; 95\% CI: 1.424 42.002), elevated serum LDH level ( $\mathrm{P}=0.046 ; \mathrm{HR}=6.510 ; 95 \%$ CI: $1.035-40.949)$, deep lesion ( $\mathrm{P}=0.005 ; \mathrm{HR}=10.957$; 95\% CI: 2.050-58.569), and CNS with systemic involvement $(\mathrm{P}=0.023$; HR=2.730; 95\% CI: 1.151-6.479) were independent poor prognostic factors for the patients. The cases with lymphocyte absolute count $>0.75 \times 10^{9} / \mathrm{L}$ (HR=0.047; $95 \%$ CI: $0.003-$ 0.732) had better prognosis. The OS of DLBCL patients with secondary CNS lymphoma was inferior to DLBCL patients without CNS involvement. There was no significant difference between the patients with CNS and extra-CNS involvement. There was no significant difference between the patients with CNS involvement and stage III-IV DLBCL cases without CNS lymphoma.

Conclusion: In conclusion, elevated serum $\mathrm{LDH}$ was independent high-risk factor for secondary CNS lymphoma. For DLBCL patients with CNS involvement, ECOG score $>2$, elevated serum LDH level, deep lesion, lymphocyte absolute count $\leq 0.75 \times 10^{9} / \mathrm{L}$ and $\mathrm{CNS}$ with systemic involvement retained a significant association with outcome.

Keywords: diffuse large B-cell lymphoma, central nervous system involvement, risk factors, prognostic

\section{Introduction}

Diffuse large B-cell lymphoma (DLBCL) is the most common pathological type of non-Hodgkin lymphoma. ${ }^{1}$ Although monoclonal anti-CD20 antibody rituximab 
(R) combined with chemotherapy improves the remission rate and prolongs overall survival (OS), secondary central nervous system (SCNS) involvement in DLBCL (including relapse or progression) seriously affects the efficacy of treatments for DLBCL. ${ }^{2,3}$ In the past literature, the incidence of CNS involvement is about $5-20 \%{ }^{3,4}$ Age $>60$ years, elevated serum $\mathrm{LDH}$, high international prognostic index (IPI), Ann Arbor stage III-IV, involment of extranodal sites, and involvement of specifically extranodal sites which contains kidney, nasopharynx, adrenal glands, bone marrow, breast or testes are the most frequently reported high risk factors of CNS involvement. $^{3,5-10}$ In particular, patients with double expresser (DE) or double hit (DH) are at heightened risk for CNS relapse/progression. ${ }^{11,12,14,15}$ Recent studies have shown that the CNS-IPI was a new risk model for CNS relapse in patients with DLBCL. ${ }^{13,16,17}$ Whether rituximab combined with chemotherapy and prophylactic intrathecal therapy can reduce the risk of CNS involvement remains controversial. A few studies have shown that the use of rituximab can reduce the incidence of CNS. But this conclusion is currently controversial. ${ }^{3,4,7,9}$ Few studies have reported whether the use of liposomal doxorubicin can reduce the occurrence of CNS involvement of DLBCL. Some researchers have reported that prophylactic treatment may reduce the recurrence of CNS relapse, prophylactic treatment strategies contain intrathecal injection (IT) chemotherapy alone and highdose IV MTX and/or cytarabine. ${ }^{18}$ However, the optimal prophylactic strategy remains unclear. Early identification and screening for CNS involvement risk factors with close follow-up or early prevention are expected to improve both the efficacy of treatments for and the prognosis of this disease. ${ }^{19-21}$

Due to rapid tumor growth and lack of effective treatment strategies, the prognosis of DLBCL patients with CNS involvement is very poor, and the median survival time is approximately 2 to 6 months. ${ }^{5}$ Longtime survivors are rarely observed. Therefore, the treatment for DLBCL with CNS involvement is in urgent need of improvement. However, there were few studies reported the prognostic factors for DLBCL patients with CNS involvement. There is no effective prognostic model for the disease.

The aim of this study was to reveal the risk factors associated with CNS involvement in patients with DLBCL and to explore prognostic for DLBCL patients with CNS involvement. A total of 38 DLBCL patients with CNS involvement were admitted to our hospital, and their clinical characteristics, risk factors, and prognosis were analyzed.

\section{Materials and Methods \\ Patients and Clinical Data}

A total of 138 DLBCL patients were recruited at our center from January 1, 2013 to June 31, 2018. All patients were followed up until 31 December 2018. In a total of 138 patients, there were 38 patients had CNS involvement. Among these 38 patients, 15 patients exhibited CNS involvement while DLBCL was pathologically confirmed 15 patients exhibited CNS involvement at initial diagnosis of DLBCL, and 23 patients developed CNS involvement during or after initial chemotherapy. When we analyzed the risk factors of CNS involvement in DLBCL patients, 15 patients with CNS involvement at the time of diagnosis were excluded. All cases in initial diagnosis of DLBCL were based on the pathological diagnosis of lymph node or organ biopsy.

\section{Diagnosis of CNS Involvement}

CNS involvement was determined according to the findings of magnetic resonance imaging (MRI) or cerebrospinal fluid tests together with symptoms of CNS. For patients with suspected CNS lymphoma, biopsy or surgical resection should be performed for pathological confirmation, if possible. CNS involvement was limited to the eyes, leptomeningeal, spinal cord and brain parenchyma. Epidural involvement was excluded from the CNS manifestation.

\section{Treatment of Newly Diagnosed DLBCL}

All patients newly diagnosed with DLBCL who accepted the CHOP (cyclophosphamide, doxorubicin, vincristine, and prednisone) or R-CHOP standard chemotherapy regimens were treated according to the patient's wishes.

\section{Statistical Analysis}

We used descriptive statistics to summary the patient's clinical characteristics. Qualitative data were compared by the chi-square test, and the strength of risk factors was calculated as the odds ratio (OR) and corresponding 95\% CI. Univariate survival analysis was performed with the log rank test. A Cox regression model was used for multivariate analysis of prognosis. A KaplanMeier curve was used to calculate the progression-free survival and overall survival durations. P-values less 
than 0.05 were considered statistically significant. All data were analyzed using the Stata 12.0 software package.

\section{Results}

\section{Baseline Characteristics of 123 Patients Without CNS Involvement at Diagnosis}

None of the 123 patients without CNS involvement at diagnosis presented CNS involvement. The clinical characteristics of 123 DLBCL patients, including 65 males and 58 females, were examined. The median age of initial DLBCL onset was 54 (41-85) years. The ECOG score was $0-2$ in 96 cases (78\%) and 3-4 in 27 cases $(22 \%)$. Seventeen patients $(13.8 \%)$ were diagnosed with DLBCL as a bulky disease. Thirty-three patients $(26.8 \%)$ suffered from extranodal site involvement (excluding the CNS) at the time of onset. At onset, 57 patients $(46.3 \%)$ were stage III-IV, and 66 patients $(53.7 \%)$ were stage I-II. The IPI was $>2$ in 53 patients (43.1\%) and $\leq 2$ in the remaining $70(56.9 \%)$ patients at onset. The LDH level was higher than normal in 42 patients $(34.1 \%)$. Thirty-five patients $(43 \%)$ had germinal center B-cell-like (GCB) disease, 52 patients (30\%) had non-GCB disease, and the pathological type in the remaining 36 patients was unclassified. Bone marrow involvement occurred in 17 patients (13.8\%). A total of 100 patients $(81.3 \%)$ were treated with rituximab and 42 patients $(34.1 \%)$ were treated with doxorubicin liposome. We performed CNS-IPI scores on these 123 patients, the model defining the CNS-IPI consists of age $>60$ years, LDH $>$ normal, ECOG $>1$, Stage III/IV disease, extranodal involvement, kidney and/or adrenal glands involved. Each factor scored 1 point if present or 0 if not present. Patients scoring 0 to 1 point formed the low-risk group ( $\mathrm{n}=49,39.9 \%)$, patients scoring 2 to 3 points formed the intermediate-risk group $(\mathrm{n}=56$, $45.5 \%$ ), and patients scoring 4 to 6 points formed the high-risk group ( $\mathrm{n}=18$ 14.6\%). The respective 2-year rates for the development of CNS disease were $14.3 \%$ for the low-risk group, $17.6 \%$ for the intermediate-risk group, and $33.3 \%$ for the high-risk group.

\section{Univariate and Multivariate Analysis of CNS Involvement Risk Factors}

Of the 123 cases with DLBCL, 23 patients developed CNS involvement, some due to disease progression and some due to relapse. The incidence of CNS involvement was $18.7 \%$. All patients were analyzed as shown in the following table (Table 1). Several factors were analyzed, including age, gender, ECOG score, bulky disease, extranodal site involvement, Ann Arbor stage, IPI, serum LDH, GCB or Non-GCB pathological type, bone marrow involvement, prophylactic intrathecal injection therapy, whether use rituximab and doxorubicin liposome, etc. Univariate analysis showed that ECOG score $>2$ $(\mathrm{P}=0.006 ; \mathrm{OR}=3.756), \mathrm{IPI}>2 \quad(\mathrm{P}=0.005 ; \mathrm{OR}=3.892), \mathrm{Ann}$ Arbor stage III-IV $(\mathrm{P}=0.003 ; \mathrm{OR}=4.25)$, elevated serum LDH level $(\mathrm{P}=0.012 ; \mathrm{OR}=3.183)$ were high-risk factors for DLBCL patients developing CNS involvement. Use of doxorubicin liposome $(\mathrm{P}=0.018 ; \mathrm{OR}=0.235)$ was a protective factor for DLBCL patients developing CNS involvement. The other factors, including age, gender, bulky disease, extranodal sites involvement, bone marrow involvement, GCB or Non-GCB pathological type and rituximab use before CNS involvement were not predictive of CNS involvement by univariate analysis. Multivariate analysis identified $\mathrm{LDH}(\mathrm{P}=0.030$; $\mathrm{HR}=4.035$; 95\% CI: 1.147-14.195) was an independent predictor of CNS involvement (Table 2).

\section{Baseline Characteristics, Treatment, and Outcome of 38 DLBCL Patients with CNS Involvement}

Table 3 shows that the baseline characteristics of 38 DLBCL patients with CNS involvement. From the total of 38 patients of DLBCL with CNS involvement, 15 patients had CNS involvement at initial diagnosis of DLBCL, and 23 patients were diagnosed with CNS involvement during or after first-line chemotherapy. Isolated CNS involvement occurred in 11 patients, while CNS involvement plus systemic disease occurred in 27 patients. First-line treatment for DLBCL patients with CNS involvement was different, which included high-dose methotrexate (HD-MTX) only $(\mathrm{n}=3)$, MTX combined CHOP $(n=11)$, MTX combined R-CHOP $(n=13)$, CHOP $(n=4)$, DHAP (Dexamethasone, Cisplatin, Cytarabine) $(n=2)$, MTX combined Idarubicin (IDA) $(n=2)$, whole-brain radiotherapy (WBRT) $(n=2)$, no treatment $(n=1)$. For patients with leptomeningeal abnormal, lumbar puncture and intrathecal injection chemotherapy were given as routine treatment. In our center, we often intrathecal injection MTX, cytarabine (Ara-C) and dexamethasone $(n=17)$. In the patients treated with HD-MTX, we used the MTX dose was $3.5-8 \mathrm{~g} / \mathrm{m}^{2}$. After 2-4 cycles of chemotherapy or one 
Table I Univariate Analysis of Risk Factors for CNS Involvement

\begin{tabular}{|c|c|c|c|c|c|c|}
\hline Clinical Features & CNS (n) & Non-CNS (n) & OR & $95 \% \mathrm{Cl}$ & $\chi^{2}$ & P Value \\
\hline Gender & & & \multirow{3}{*}{1.875} & \multirow[t]{3}{*}{$(0.70 \mathrm{I}-4.773)$} & \multirow[t]{3}{*}{1.738} & \multirow[t]{3}{*}{0.187} \\
\hline Male & 15 & 50 & & & & \\
\hline Female & 8 & 50 & & & & \\
\hline Age (years) & & & \multirow{3}{*}{1.034} & \multirow[t]{3}{*}{$(0.419-2.480)$} & \multirow[t]{3}{*}{0.005} & \multirow[t]{3}{*}{0.943} \\
\hline$\geq 60$ & 11 & 47 & & & & \\
\hline$<60$ & 12 & 53 & & & & \\
\hline ECOG Score & & & 3.756 & $(1.466-10.22)$ & 7.652 & 0.006 \\
\hline$>2$ & 10 & 17 & & & & \\
\hline$\leq 2$ & 13 & 83 & & & & \\
\hline Bulky Disease & & & & $(0.022-1.393)$ & 2.132 & 0.144 \\
\hline Yes & 1 & 16 & 0.239 & & & \\
\hline No & 22 & 84 & & & & \\
\hline Extranodal Site Involvement & & & 1.6 & $(0.599-4.054)$ & 0.912 & 0.340 \\
\hline$\geq 2$ & 8 & 25 & & & & \\
\hline$<2$ & 15 & 75 & & & & \\
\hline Stage & & & 4.25 & $(1.6 \mid 4-11.56)$ & 8.649 & 0.003 \\
\hline III--IV & 17 & 40 & & & & \\
\hline I-II & 6 & 60 & & & & \\
\hline IPI & & & 3.892 & $(1.406-9.766)$ & 8.087 & 0.005 \\
\hline$>2$ & 16 & 37 & & & & \\
\hline$\leq 2$ & 2 & 63 & & & & \\
\hline $\mathrm{LDH}>236 \mathrm{U} / \mathrm{L}$ & & & 3.183 & $(1.299-8.026)$ & 6.299 & 0.012 \\
\hline Yes & 13 & 29 & & & & \\
\hline No & 10 & 71 & & & & \\
\hline GCB/Non-GCB & & & 0.516 & $(0.140-1.936)$ & 0.879 & 0.348 \\
\hline GCB & 3 & 32 & & & & \\
\hline Non-GCB & 8 & 44 & & & & \\
\hline Bone marrow Involvement & & & 1.409 & $(0.459-4.529)$ & 0.303 & 0.582 \\
\hline Yes & 4 & 13 & & & & \\
\hline No & 19 & 87 & & & & \\
\hline Rituximab & & & 0.898 & $(0.303-2.974)$ & 0.032 & 0.858 \\
\hline YES & 19 & 81 & & & & \\
\hline No & 4 & 19 & & & & \\
\hline Dorubicin Liposome & & & 0.235 & $(0.070-0.806)$ & 5.603 & 0.018 \\
\hline Yes & 3 & 39 & & & & \\
\hline No & 20 & 61 & & & & \\
\hline
\end{tabular}

cycle of radiotherapy, 23 patients showed rapid progression. We administered second-line treatment, i.e., DHAP, ICE (Ifosfamide, Carboplatin, Etoposide), Ara-C combined with temozolomide (TMZ), and WBRT. In addition, there were four patients treated with autologous hematopoietic stem-cell transplantation (ASCT).
In total, the median follow-up time was 2 years (range from 0.5 to 5.5 years). Fifteen patients (39.5\%) died, 13 patients $(34.2 \%)$ were in complete remission (CR), 3 patients $(7.9 \%)$ were in progression disease (PD), 5 patients (13.2\%) were in partial remission (PR), and 2 patients (5.2\%) were in stable disease (SD). The median PFS time 
Table 2 Multivariate Analysis of Risk Factors for CNS Involvement

\begin{tabular}{|l|l|l|}
\hline Clinical Factors & P Value & Hazard Ratio (95\% CI) \\
\hline Gender(male/female) & 0.186 & $2.173(0.688-6.862)$ \\
Age $\geq 60$ years & $0.4 I I$ & $0.605(0.183-2.003)$ \\
ECOG >2 & $0.11 \mathrm{II}$ & $3.166(0.767-13.057)$ \\
IPI >2 & 0.084 & $4.564(0.815-25.547)$ \\
Elevated LDH & 0.030 & $4.035(1.147-14.195)$ \\
Stage I-II/III-IV & 0.859 & $0.861(0.164-4.522)$ \\
Extranodal involvement $\geq 2$ & 0.959 & $1.038(0.245-4.398)$ \\
Bulky disease & 0.059 & $0.113(0.012-1.088)$ \\
No use of dorubicin liposome & $0.05 \mathrm{I}$ & $0.24 \mathrm{I}(0.058-1.004)$ \\
\hline
\end{tabular}

Table 3 Baseline Characteristics of 38 DLBCL Patients with CNS Involvement

\begin{tabular}{|c|c|}
\hline Baseline Characteristics (at Time of Diagnosis) & n (\%) \\
\hline Age (years) & $59.84 \pm 8.71^{\mathrm{a}}$ \\
\hline \multicolumn{2}{|l|}{ Gender } \\
\hline Male & $26(68.4 \%)$ \\
\hline Female & $12(2 \mid .6 \%)$ \\
\hline \multicolumn{2}{|l|}{$\mathrm{LDH}$} \\
\hline High (>236U/L) & $15(42.9 \%)$ \\
\hline Normal ( $\leq 236 \mathrm{U} / \mathrm{L})$ & $23(57.1 \%)$ \\
\hline \multicolumn{2}{|l|}{ Bulky Disease ${ }^{b}$} \\
\hline Yes & $9(7.1 \%)$ \\
\hline No & $29(92.9 \%)$ \\
\hline \multicolumn{2}{|l|}{ Sites } \\
\hline CNS only & II(35.7\%) \\
\hline CNS and Systemic & $27(64.3 \%)$ \\
\hline \multicolumn{2}{|l|}{ Deep lesion ${ }^{c}$} \\
\hline Yes & $2 \mathrm{I}(55.3 \%)$ \\
\hline No & $17(44.7 \%)$ \\
\hline \multicolumn{2}{|l|}{ CSF Protein } \\
\hline$>1.0 \mathrm{~g} / \mathrm{L}$ & $13(43.3 \%)$ \\
\hline$\leq \mathrm{I} .0 \mathrm{~g} / \mathrm{L}$ & $17(66.7 \%)$ \\
\hline \multicolumn{2}{|l|}{ CSF Nuclear Cells } \\
\hline$>8 \times 10^{6} / \mathrm{L}$ & $10(32.3 \%)$ \\
\hline$\leq 8 \times 10^{6} / \mathrm{L}$ & $21(67.7 \%)$ \\
\hline \multicolumn{2}{|l|}{ CSF Lymphoma Cells } \\
\hline Positive & $24(35.7 \%)$ \\
\hline Negative & $14(64.3 \%)$ \\
\hline \multicolumn{2}{|l|}{ Eyes Involvement } \\
\hline Yes & $7(7.1 \%)$ \\
\hline No & $3 \mathrm{I}(92.9 \%)$ \\
\hline
\end{tabular}

Notes: ${ }^{\mathrm{a}}$ Mean \pm SD; ${ }^{\mathrm{b}}$ Bulky disease refers to the lesion diameter greater than $3 \mathrm{~cm}$; 'Deep lesions defined as lesions located more than $3 \mathrm{~cm}$ from the brain surface.

after-CNS involvement was 12.5 months. The median OS time after-CNS involvement was 22 months.

\section{Analysis of Prognostic Factors at the Time of CNS Involvement}

Univariate analysis by log rank test and multivariate analysis by cox multiple regression were performed to analyze prognostic factors among 38 patients of DLBCL with CNS involvement. Univariate prognostic analysis showed that ECOG score $>2 \quad(\mathrm{P}=0.002 ; \mathrm{HR}=5.215 ; 95 \%$ CI: $1.842-$ 14.76), cerebrospinal fluid (CSF) protein $>1.0 \mathrm{~g} / \mathrm{L}(\mathrm{P}=0.004$; $\mathrm{HR}=10.84 ; 95 \%$ CI: 2.165-54.32), lymphocyte absolute count $\leq 0.75 \times 10^{9} / \mathrm{L}(\mathrm{P}=0.023$; HR $=8.857 ; 95 \%$ CI: 1.999 $39.25)$ and elevated $\mathrm{LDH}$ level $(\mathrm{P}=0.005 ; \mathrm{HR}=5.355 ; 95 \%$ CI: 1.648-17.4) were poor prognostic factors (Table 4). Multivariate prognostic analysis identified ECOG score $>2$ ( $\mathrm{P}=0.018 ; \mathrm{HR}=7.333 ; 95 \%$ CI: $1.424-42.002)$, elevated LDH level ( $\mathrm{P}=0.046$; HR=6.510; 95\% CI: $1.035-40.949)$, deep lesion (defined as lesions located more than $3 \mathrm{~cm}$ from the brain surface) $(\mathrm{P}=0.005$; $\mathrm{HR}=10.957 ; 95 \% \mathrm{CI}: 2.050$ 58.569), and $\mathrm{CNS}$ with systemic involvement $(\mathrm{P}=0.023$; $\mathrm{HR}=2.730$; 95\% CI: $1.151-6.479)$ were independent poor prognostic factors. Lymphocyte absolute count $>0.75 \times 10^{9} / \mathrm{L}$ $(\mathrm{P}=0.029 ; \mathrm{HR}=0.047 ; 95 \%$ CI: 0.003-0.732) was protective prognostic factor (Table 5). Other factors, such as gender, age $\geq 60$ years, bulky disease, CSF nuclear cells, neutrophil absolute count $<6.3 \times 10^{9} / \mathrm{L}$, peripheral blood white blood cell (WBC) and site of CNS involvement had no impact on prognosis $(\mathrm{P}>0.05)$. We use these five factors $(\mathrm{ECOG}>2$, elevated LDH level, deep lesion, CNS with systemic involvement, lymphocyte absolute count $\leq 0.75 \times 10^{9} / \mathrm{L}$ ) established a simple prognostic scoring system, with 1 point with each term. Patients scoring 0 to 1 point formed the low-risk group ( $\mathrm{n}=11,28.9 \%$ ), patients scoring 2 to 3 points formed the intermediate-risk group $(\mathrm{n}=20,52.7 \%)$ and patients scoring 4 to 5 points formed the high-risk group $(\mathrm{n}=7,18.4 \%)$, with 1-year survival rate of $90.9 \%(10 / 11)$, $40 \%(8 / 20)$, and $14.3 \%(1 / 7)$ respectively.

\section{Kaplan-Meier Curve Estimation of PFS and OS of DLBCL Patients with and Without CNS Involvement}

The result revealed that the median PFS and OS durations of DLBCL patients after CNS involvement were 12.5 months and 22 months, respectively (Figures 1 and 2). As shown in our research, the overall survival of DLBCL patients with CNS involvement (SCNSL) was poorer than DLBCL patients without $\mathrm{CNS}$ involvement $(\mathrm{P}=0.032 ; \mathrm{HR}=2.282$; 95\% CI: 1.075-4.842) (Figure 3). It is notable that there was no difference observed between the cases with CNS 
Table 4 Univariate Analysis of 38 DLBCL Patients with CNS Involvement

\begin{tabular}{|c|c|c|c|c|c|c|}
\hline Clinical Factors & Median Survival (Months) & Number & Death (N) & HR 95\% Cl & $\chi^{2}$ & $P$ Value \\
\hline Gender & & & & $1.063(0.332-3.399)$ & 0.011 & 0.918 \\
\hline Male & 22 & 26 & II & & & \\
\hline Female & NR & 12 & 4 & & & \\
\hline Age (years) & & & & $2.683(0940-7.663)$ & 3.399 & 0.065 \\
\hline$\geq 60$ & 21 & 21 & II & & & \\
\hline$<60$ & NR & 17 & 4 & & & \\
\hline ECOG Score & & & & $5.215(1.842-14.76)$ & 9.678 & 0.002 \\
\hline$>2$ & 8 & 19 & 13 & & & \\
\hline$\leq 2$ & NR & 19 & 2 & & & \\
\hline $\mathrm{LDH}>236 \mathrm{U} / \mathrm{L}$ & & & & $5.355(\mid .648-17.4)$ & 7.789 & 0.005 \\
\hline Yes & 8 & 15 & 9 & & & \\
\hline No & NR & 23 & 6 & & & \\
\hline CSF Protein & & & & $10.84(2.165-54.32)$ & 8.406 & 0.004 \\
\hline$\geq 1.0 \mathrm{~g} / \mathrm{L}$ & 8 & 13 & 6 & & & \\
\hline$<1.0 \mathrm{~g} / \mathrm{L}$ & NR & 17 & 7 & & & \\
\hline CSF Nuclear Cell & & & & $2.254(0.782-6.495)$ & 2.265 & 0.132 \\
\hline$>8 \times 10^{6} / \mathrm{L}$ & 21 & 24 & II & & & \\
\hline$\leq 8 \times 10^{6} / \mathrm{L}$ & NR & 14 & 4 & & & \\
\hline Lymphocyte Count & & & & $8.857(1.999-39.25)$ & 5.025 & 0.023 \\
\hline$\leq 0.75 \times 10^{9} / \mathrm{L}$ & 3.5 & 4 & 2 & & & \\
\hline$>0.75 \times 10^{9} / \mathrm{L}$ & 31 & 34 & 13 & & & \\
\hline White Blood Cell & & & & & & \\
\hline $3.5-9.5 \times 10^{9} / \mathrm{L}$ & NR & 21 & 7 & & 1.586 & 0.452 \\
\hline$>9.5 \times 10^{9} / \mathrm{L}$ & 21 & 10 & 4 & & & \\
\hline$<3.5 \times 10^{9} / \mathrm{L}$ & 14 & 7 & 4 & & & \\
\hline Neutrophil Count & & & & & & \\
\hline $1.8-6.3 \times 10^{9} / \mathrm{L}$ & NR & 24 & 12 & & 3.202 & 0.074 \\
\hline$>6.3 \times 10^{9} / \mathrm{L}$ & NR & 11 & 3 & & & \\
\hline$<1.8 \times 10^{9} / \mathrm{L}$ & 21 & 3 & 0 & & & \\
\hline Neutrophil (\%) & & & & $1.269(0.424-3.793)$ & 0.182 & 0.670 \\
\hline$\geq 75 \%$ & NR & 16 & 6 & & & \\
\hline$<75 \%$ & 22 & 22 & 9 & & & \\
\hline Lymphocyte (\%) & & & & $2.483(0.862-7.153)$ & 2.837 & 0.092 \\
\hline$<20 \%$ & 14 & 21 & 10 & & & \\
\hline $20-50 \%$ & NR & 17 & 5 & & & \\
\hline Bulky Disease & & & & $1.476(0.47-4.635)$ & 0.254 & 0.614 \\
\hline Diameter $\geq 3 \mathrm{~cm}$ & 21 & 9 & 4 & & & \\
\hline Diameter $<3 \mathrm{~cm}$ & 31 & 29 & II & & & \\
\hline Deep Lesion & & & & $2.745(0.960-7.852)$ & 3.546 & 0.060 \\
\hline Yes & 21 & 21 & 12 & & & \\
\hline No & NR & 17 & 3 & & & \\
\hline Systemic Involvement with SCNS & & & & I.357(0.4I0-4.488) & 0.250 & 0.617 \\
\hline Yes & 22 & 27 & 12 & & & \\
\hline No & NR & 11 & 3 & & & \\
\hline
\end{tabular}

(Continued) 
Table 4 (Continued).

\begin{tabular}{|l|l|l|l|l|l|l|}
\hline Clinical Factors & Median Survival (Months) & Number & Death (N) & HR 95\% Cl & $\chi^{2}$ & P Value \\
\hline Site of CNS Involvement & & & & & 4.931 \\
Parenchymal (incl. eye) & NR & 21 & 6 & 0.085 \\
Leptomeningeal Only & 8 & 2 & 1 & & \\
Both & 8 & 15 & 8 & & \\
\hline
\end{tabular}

Table 5 Multivariate Analysis of 38 DLBCL Patients with CNS Involvement

\begin{tabular}{|c|c|c|c|c|c|}
\hline Clinical Factors & Std. Err. & Z Value & $P$ Value & HR & $95 \% \mathrm{Cl}$ \\
\hline Age $\geq 60$ years & 0.861 & 0.053 & 0.951 & 1.054 & $(0.195-5.694)$ \\
\hline $\mathrm{ECOG}>2$ & 0.863 & 2.045 & 0.018 & 7.333 & $(1.424-42.002)$ \\
\hline $\mathrm{LDH}>236 \mathrm{U} / \mathrm{L}$ & 0.938 & 1.873 & 0.046 & 6.510 & $(1.035-40.949)$ \\
\hline CSF protein $>1.0 \mathrm{~g} / \mathrm{L}$ & 0.507 & 0.642 & 0.206 & 1.900 & $(0.703-5.133)$ \\
\hline Lymphocyte $>0.75 \times 10^{9} / \mathrm{L}$ & 1.406 & -3.067 & 0.029 & 0.047 & $(0.003-0.732)$ \\
\hline Lymphocyte $(\%)<20 \%$ & 0.899 & -0.308 & 0.732 & 0.735 & $(0.126-4.279)$ \\
\hline Deep lesion & 0.855 & 2.395 & 0.005 & 10.957 & $(2.050-58.569)$ \\
\hline Neutrophil count $<6.3109 / \mathrm{L}$ & 1.025 & -1.078 & 0.293 & 0.340 & $(0.046-2.538)$ \\
\hline Site of Involvement (CNS + Systemic) & $0.44 I$ & 1.004 & 0.023 & 2.730 & $(1.15 I-6.479)$ \\
\hline
\end{tabular}

only and extra-CNS involvement $(\mathrm{P}=0.181 ; \mathrm{HR}=0.482 ; 95 \%$ CI: $0.165-1.405)$. There was no difference observed between the patients with CNS involvement at the time of DLBCL diagnosed and during or after first-line therapy ( $\mathrm{P}=0.973$; $\mathrm{HR}=1.182$; 95\% CI: 0.429-3.259). There was no significant difference between the patients with CNS involvement and stage III-IV DLBCL cases without CNS involvement $(\mathrm{P}=0.238 ; \mathrm{HR}=1.555 ; 95 \% \mathrm{CI}$ : 0.747-3.238) (Figures 4-6)

\section{Discussion}

The incidence of CNS involvement in DLBCL ranges from $5 \%$ to $20 \%,{ }^{3,4}$ at $10 \%$ in Asian countries and $5 \%$ in

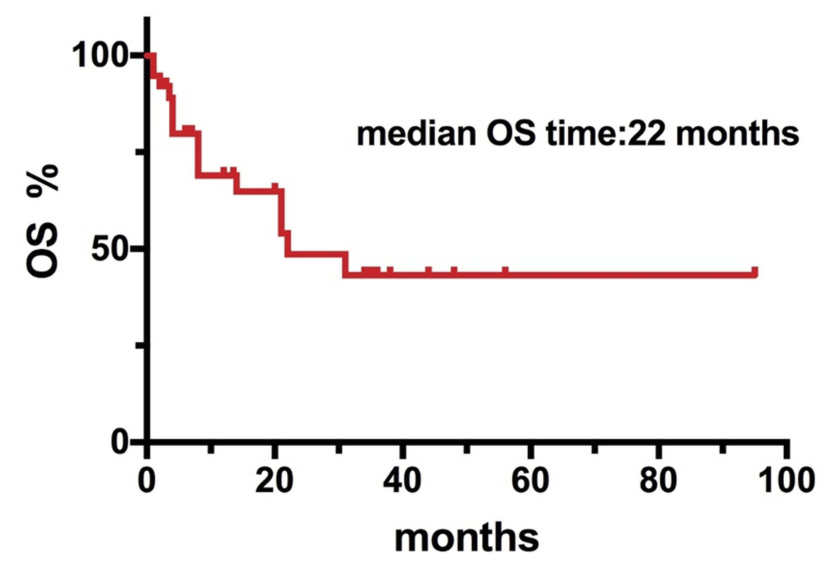

Figure I Kaplan-Meier curve of the median OS durations of $38 \mathrm{DLBCL}$ patients after CNS involvement.
European countries. CNS involvement in DLBCL mainly occurs within less than 1 year after diagnosis, after a median of 6 months. ${ }^{9,22,23}$ CNS involvement in DLBCL is divided into the following three scenarios: (1) patients with systemic remission, simple recurrence of CNS involvement; (2) patients achieve remission after treatment, but systemic recurrence with CNS involvement occurs at the same time; and (3) patients develop CNS involvement early in the treatment period (within 6 months). The CNS involvement can manifest in the brain parenchyma, leptomeningeal, spinal cord and eyes. Therefore, DLBCL patients with suspected CNS involvement should be examined by

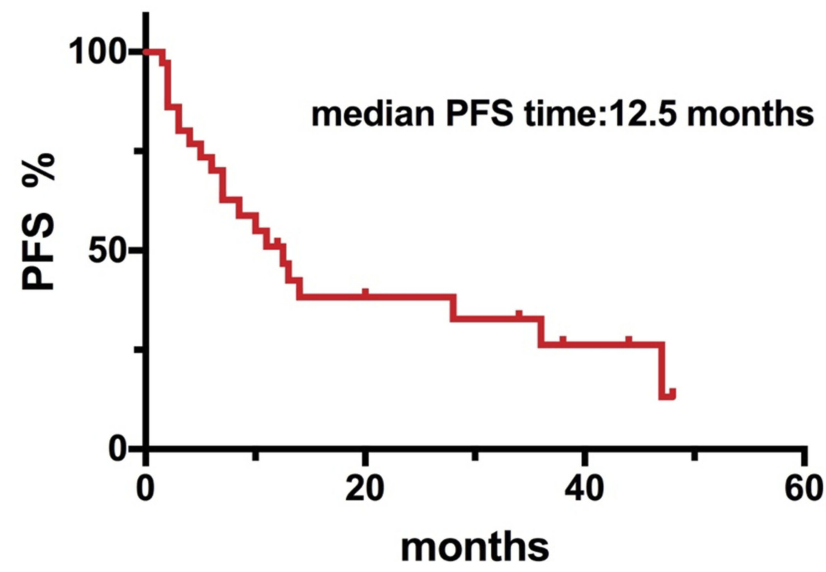

Figure 2 Kaplan-Meier curve of the median PFS durations of 38 DLBCL patients after CNS involvement. 


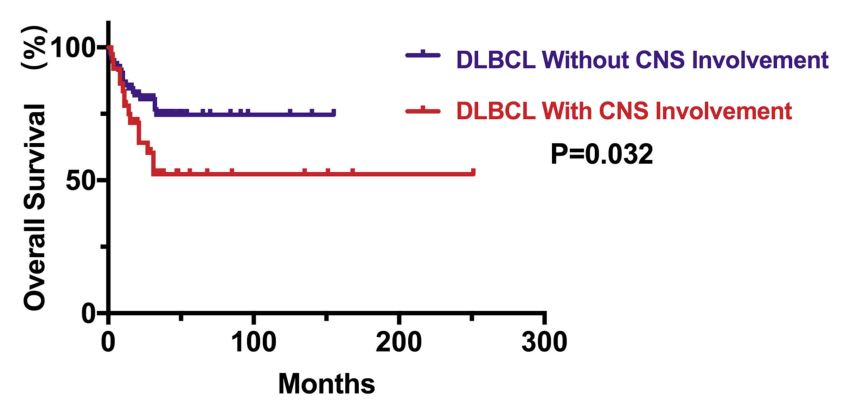

Figure 3 Kaplan-Meier curve of OS time between 38 DLBCL patients with CNS involvement (SCNSL) and 100 DLBCL patients without CNS involvement.

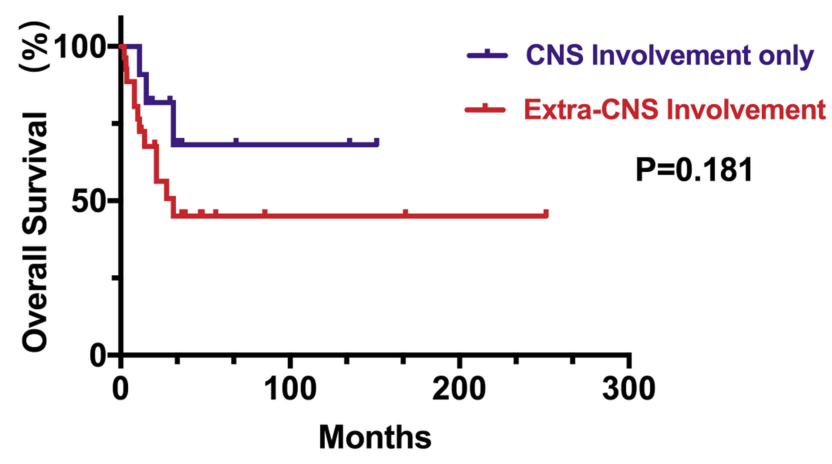

Figure 4 Kaplan-Meier curve of OS time between II DLBCL patients with CNS involvement only and $27 \mathrm{DLBCL}$ patients with extra-CNS involvement.

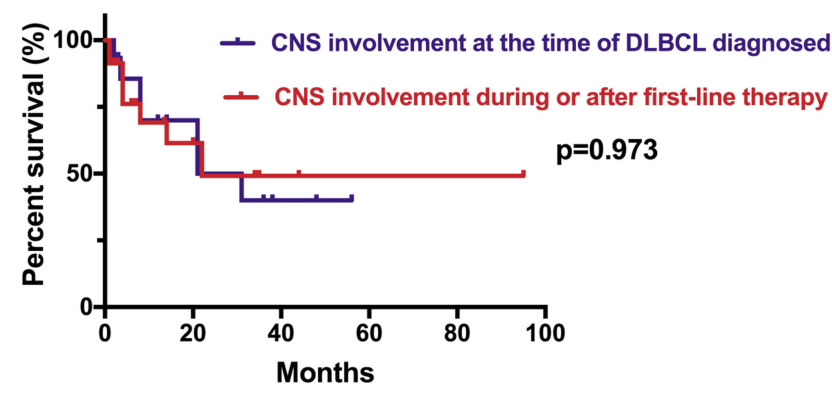

Figure 5 Kaplan-Meier curve of OS time between the 15 patients with CNS involvement at the time of DLBCL diagnosed and 23 patients with CNS involvement during or after first-line therapy.

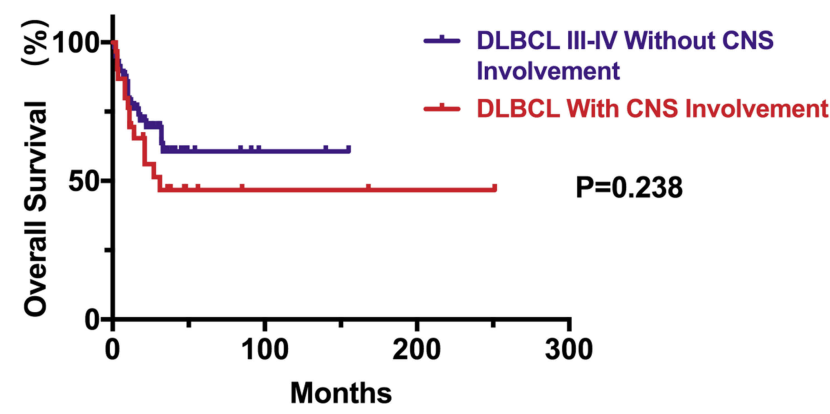

Figure 6 Kaplan-Meier curve of OS time between the 38 patients with CNS involvement and 40 stage III-IV DLBCL cases without CNS involvement. cerebrospinal fluid cytology, contrast-enhanced head and spinal cord MRI and eye Doppler ultrasound. If necessary and possible, diagnostic vitrectomy and brain biopsy should be performed to confirm the diagnosis. The incidence of CNS involvement in this study was higher than that previously reported in European and American countries. This discrepancy may be related to race, the higher proportion of selected patients in the high-risk group and the longer follow-up period of our study. Previous studies have reported that age $>60$ years, high LDH, ECOG score $>2$, IPI $>2$, multiple extranodal site involvement, bone marrow involvement, and Ann Arbor stage III-IV are risk factors of CNS involvement. ${ }^{5-10}$ In our study, univariate analysis showed that ECOG score $>2$, IPI $>2$, Ann Arbor stage IIIIV, elevated serum LDH level were high-risk factors for DLBCL patients developing CNS involvement. Compared with previous related studies, our study identified that elevated serum LDH was the independent high-risk factor of CNS involvement from multivariate analysis. Other studies have shown that the involvement of special anatomical sites, such as the breasts, testes, nasopharynx, adrenal glands, and bone marrow are risk factors of CNS involvement. ${ }^{9,10}$ In particular, patients with $\mathrm{DE}$ or DH are at heightened risk for CNS relapse/ progression. ${ }^{11,12,14,15}$ Recent studies have shown that the CNS-IPI was a new risk model for CNS relapse in patients with DLBCL. ${ }^{13,16,17}$ We performed CNS-IPI scores on these 123 patients: patients scoring 0 to 1 point formed the low-risk group ( $\mathrm{n}=49,39.9 \%)$, patients scoring 2 to 3 points formed the intermediate-risk group $(\mathrm{n}=56,45.5 \%)$, and patients scoring 4 to 6 points formed the high-risk group $(\mathrm{n}=1814.6 \%)$. The respective 2-year rates for the development of CNS disease were $14.3 \%$ for the low-risk group, $17.6 \%$ for the intermediate-risk group, and $33.3 \%$ for the high-risk group. Whether rituximab combined with chemotherapy and prophylactic intrathecal therapy can reduce the risk of CNS involvement remains controversial. A few studies have shown that the use of rituximab can reduce the incidence of CNS. But This conclusion is currently controversial. ${ }^{3,4,7,9}$ We found that the use of rituximab did not prevent CNS involvement. This conclusion may be related to the limitations of selection bias in retrospective studies. Few studies have reported whether the use of liposomal doxorubicin can reduce the occurrence of CNS involvement of DLBCL. In our study, univariate analysis showed that the use of liposomal doxorubicin can protect DLBCL patients from CNS involvement. However, the data were limited by the retrospective nature and warrant 
confirmation in prospective randomized studies. Some researchers have reported that prophylactic treatment may reduce the recurrence of CNS relapse, prophylactic treatment strategies contain IT chemotherapy alone and highdose IV MTX and/or cytarabine. ${ }^{18}$ However, the optimal prophylactic strategy remains unclear. Since our patients did not routinely receive prophylactic treatment and we did not take double-expresser or double-hit test for every patients, we did not analyze the effect of prophylactic therapy and double expresser or double hit on CNS involvement. This part of conclusion is lacking.

Previous studies have shown that DLBCL patients with CNS involvement have a poor prognosis, with a median OS duration of 2-6 months. ${ }^{5}$ We found that the median PFS and OS durations of DLBCL patients after CNS involvement were 12.5 months and 22 months, respectively (Fig 1-2). Univariate analysis and multivariate analysis were performed to analyze prognostic factors among 38 patients of DLBCL with CNS involvement. The univariate prognostic analysis showed that ECOG score $>2$, CSF protein $>1.0 \mathrm{~g} / \mathrm{L}$, lymphocyte absolute count $\leq 0.75 \times 10^{9} / \mathrm{L}$ and elevated $\mathrm{LDH}$ level were poor prognostic factors (Table 4 ). The multivariate prognostic analysis identified ECOG score $>2$, elevated LDH level, deep lesion, and CNS with systemic involvement were independent poor prognostic factors. Lymphocyte absolute count $>0.75 \times 10^{9} / \mathrm{L}$ was the protective prognostic factor (Table 5). We use these five factors $(\mathrm{ECOG}>2$, elevated $\mathrm{LDH}$ level, deep lesion, CNS with systemic involvement, lymphocyte absolute count $\leq 0.75 \times 10^{9} / \mathrm{L}$ ) established a simple prognostic scoring system, with 1 point with each term. Patients scoring 0 to 1 point formed the low-risk group $(n=11$, $28.9 \%$ ), patients scoring 2 to 3 points formed the intermediate-risk group $(n=20,52.7 \%)$ and patients scoring 4 to 5 points formed the high-risk group $(n=7,18.4 \%)$, with 1-year survival rate of $90.9 \%(10 / 11), 40 \%(8 / 20)$ and $14.3 \%(1 / 7)$ respectively. However, the data were limited by the retrospective nature and warrant confirmation in prospective randomized studies.

As shown in our research, the prognosis of DLBCL patients with CNS involvement was poorer than DLBCL patients without CNS involvement (Figure 3). Unlike the previous study, our research found that there was no difference in overall survival between the cases with CNS and extra-CNS involvement. There was no difference observed between the patients with CNS involvement at the time of DLBCL diagnosed and during or after initial therapy. Meanwhile, it is notable that there was no significant difference in overall survival between the patients with CNS involvement and stage III-IV DLBCL cases without CNS involvement (Figures 4-6).

DLBCL patients with CNS involvement can be treated with WBRT, HD-MTX, polychemotherapy and autologous HSCT. The usefulness of WBRT is limited by its toxicity, especially in older patients; its true impact on outcome remains controversial. $^{24}$ HD-MTX is often effective in cases of primary and secondary CNS lymphoma; however, it is very important to determine whether CNS involvement is sensitive to MTX. In MTX-sensitive patients, HD-MTX administration is advisable, followed by thiotepa or carmustine-based conditioning regimens and autologous HSCT. ${ }^{25}$ Other agents that cross the blood-brain barrier, such as HDcytarabine or ifosfamide, have been used in combination with HD-MTX and have shown encouraging efficacy. ${ }^{25-28}$ Other regimens, such as HD-MTX combined with IV rituximab or IV HD-cytarabine combined with oral temozolomide, may be feasible options. ${ }^{29}$ Patients with resistant lymphoma should be candidates for clinical trials or other palliative treatment. ${ }^{25,30}$ In our center, we tried to take effective measures to prolong the patients' survival time. For most part of patients with CNS involvement, we considered both CNS and systemic regimens, and selected HD-MTX $\left(3.5-8 \mathrm{~g} / \mathrm{m}^{2}\right)$ combined CHOP or R-CHOP as the first-line treatment regimen. Meanwhile, for patients with leptomeningeal abnormal, lumbar puncture and intrathecal injection chemotherapy were given as routine treatment. After 2-4 cycles of chemotherapy or one cycle of radiotherapy, 23 patients showed rapid progression. We administered second-line treatment, i.e., DHAP, ICE, Ara-C combined with TMZ, and WBRT. In addition, there were four patients treated with ASCT. In total, the median follow-up time was 2 years (0.5-5.5 years). Fifteen patients $(39.5 \%)$ died, 13 patients $(34.2 \%)$ were in complete remission (CR), 3 patients $(7.9 \%)$ were in progression disease (PD), 5 patients (13.2\%) were in partial remission (PR), and 2 patients $(5.2 \%)$ were in stable disease (SD). The overall response rate was $47.4 \%$.

\section{Conclusion}

Among DLBCL patients, elevated serum LDH was an independent high-risk factor for CNS involvement. Close attention should be paid to DLBCL patients with high-risk factors, who may require early preventive treatment. For patients with CNS involvement, ECOG score $>2$, deep lesion and CNS with systemic involvement were independent poor prognostic factors for the patients. The cases with lymphocyte absolute count $>0.75 \times 109 / \mathrm{L}$ had better prognosis. The prognosis of DLBCL patients with CNS 
involvement was inferior. The median PFS time after-CNS involvement was 12.5 months. The median OS time afterCNS involvement was 22 months. Unlike the previous study, our research found that there was no difference in overall survival between the cases with CNS and extraCNS involvement. There was no difference observed between the patients with CNS involvement at the time of DLBCL diagnosed and during or after first-line therapy. Meanwhile, it is notable that there was no significant difference in overall survival between the patients with CNS involvement and the patients of DLBCL stage IIIIV without CNS involvement.

\section{Ethics}

This research was approved by the Ethics Committee of Huashan Hospital North, Fudan University. All procedures followed were in accordance with the ethical standards of the responsible committee on human experimentation (institutional and national) and with the Helsinki Declaration of 1975 , as revised in 2008. All the patients voluntarily participated in this study and provided written informed consent. All the participants' personal information is confidential.

\section{Acknowledgments}

This retrospective study is a part of research (No.16CR2043B), which was funded by the Shanghai Shenkang Hospital Development Center: Three-year Plan for Promoting Clinical Skills and Innovations in Municipal Hospitals.

\section{Disclosure}

The authors report no conflicts of interest in this work.

\section{References}

1. Al-Hamadani M, Habermann TM, Cerhan JR, Macon WR, Maurer MJ, Go RS. Non-Hodgkin lymphoma subtype distribution, geodemographic patterns, and survival in the US: a longitudinal analysis of the National Cancer Data Base from 1998 to 2011. Am J Hematol. 2015;90:790-795. doi:10.1002/ajh.24086

2. Abramson JS. High-dose chemotherapy and autologous stem -cell transplantation for secondary central nervous system lymphoma: many are called, but few are chosen. Haematologica. 2013; 98:662-664. doi:10.3324/haematol.2013.084285

3. Zhang J, Chen B, Xu X. Impact of rituximab on incidence of and risk factors for central nervous system relapse in patients with diffuse large B-cell lymphoma: a systematic review and meta-analysis. Leuk Lymphoma. 2014;55(3):509-514. doi:10.3109/10428194.2013.811239

4. Tai WM, Chung J, Tang PL, et al. Central nervous system (CNS) relapse in diffuse large B cell lymphoma (DLBCL): pre-and post-rituximab. Ann Hematol. 2011;90:809-818. doi:10.1007/s00277-010-1150-7

5. Fletcher CD, Kahl BS. Central nervous system involvement in diffuse large B-cell lymphoma: an analysis of risks and prevention strategies in the post-rituximab era. Leuk Lymphoma. 2014;55(10):2228-2240. doi:10.3109/10428194.2013.869326
6. Van Besien K, Ha CS, Murphy S, et al. Risk factors, treatment, and outcome of central nervous system recurrence in adults with intermediate-grade and immunoblastic lymphoma. Blood. 1998;91 (4):1178-1184. doi:10.1006/bcmd.1998.0171

7. Kumar A, Vanderplas A, LaCasce AS, et al. Lack of benefit of central nervous system prophylaxis for diffuse large B-cell lymphoma in the rituximab era: findings from a large national database. Cancer. 2012;118(11):2944-2951. doi:10.1002/cncr.26588

8. Schmitz N, Zeynalova S, Glass B, et al. CNS disease in younger patients with aggressive B-cell lymphoma: an analysis of patients treated on the Mabthera International Trial and trials of the German High-Grade Non-Hodgkin Lymphoma Study Group. Ann Oncol. 2012;23(5):1267-1273. doi:10.1093/annonc/mdr440

9. Tomita N, Yokoyama M, Yamamoto W, et al. Central nervous system event in patients with diffuse large B-cell lymphoma in the rituximab era. Cancer Sci. 2012;103(2):245-251.

10. Hosein PJ, Maragulia JC, Salzberg MP, et al. A multicentre study of primary breast diffuse large B-cell lymphoma in the rituximab era. $\mathrm{Br}$ J Haematol. 2014;165(3):358-363. doi:10.1111/bjh.12753

11. Herrera AF, Mei M, Low L, et al. Relapsed or refractory double-expressor and double-hit lymphomas have inferior progression-free survival after autologous stem-cell transplantation. J Clin Oncol. 2017;35(1):24-31. doi:10.1200/JCO.2016.68.2740

12. Riedell PA, Smith SM. Double hit and double expressors in lymphoma: definition and treatment. Cancer. 2018;124:4622-4632. doi: $10.1002 /$ cncr.31646

13. Schmitz N, Zeynalova S, Nickelsen M, et al. CNS international prognostic index: a risk model for CNS relapse in patients with diffuse large B-cell lymphoma treated with R-CHOP. J Cli Oncol. 2016. doi:10.1200/JCO.2015.65.6520

14. Petrich AM, Gandhi M, Jovanovic B, et al. Impact of induction regimen and stem cell transplantation on outcomes in double-hit lymphoma: a multicenter retrospective analysis. Blood. 2014;124 (15):2354-2361. doi:10.1182/blood-2014-05-578963

15. Oki Y, Noorani M, Lin P, et al. Double hit lymphoma: the MD Anderson Cancer Center clinical experience. $\mathrm{Br} J$ Haematol. 2014;166(6):891-901.doi:10.1111/bjh.12982

16. Verrou E, Gerofotis A, Karampatzakis N, et al. CNS relapse in patients with DLBCL according to CNS-IPI score and the initial therapy. Hematol Oncol. 2017;35:353-354. doi:10.1002/hon.2439_106

17. Savage KJ, Slack GW, Mottok A, et al. Impact of dual expression of MYC and BCL2 by immunohistochemistry on the risk of CNS relapse in DLBCL. Blood. 2016;127:2182-2188. doi:10.1182/blood2015-10-676700

18. Cheah CY, Herbert KE, O'Rourke K, et al. A multicentre retrospective comparison of central nervous system prophylaxis strategies among patients with high-risk diffuse large B-cell lymphoma. Br J Cancer. 2014;111(6):1072-1079. doi:10.1038/ bjc. 2014.405

19. Feugier P, Virion JM, Tilly H, et al. Incidence and risk factors for central nervous system occurrence in elderly patients with diffuse large-B-cell lymphoma: influence or rituximab. Ann Oncol. 2004;15 (1):129-133. doi:10.1093/annonc/mdh013

20. Boehme V, Schmitz N, Zeynalova S, Loeffler M, Pfreundschuh M. CNS events in elderly patients with aggressive lymphoma treated with modern chemotherapy (CHOP-14) with or without rituximab: an analysis of patients treated in the RICOVER-60 trial of the German High-Grade Non-Hodgkin Lymphoma Study Group (DSHNHL). Blood. 2009;113(17):3896-3902. doi:10.1182/blood-20 08-10-182253

21. Villa D, Connors JM, Shenkier TN, Gascoyne RD, Sehn LH, Savage KJ. Incidence and risk factors for central nervous system relapse in patients with diffuse large B-cell lymphoma: the impact of the addition of rituximab to CHOP chemotherapy. Ann Oncol. 2009;21(5):1046-1052. doi:10.1093/annonc/mdp 432 
22. Chihara D, Oki Y, Matsuo K, et al. Incidence and risk factors for central nervous system relapse in patients with diffuse large B-cell lymphoma: analyses with competing risk regression model. Leuk Lymphoma. 2011;52 (12):2270-2275. doi:10.3109/10428194.2011.596966

23. Siegal T, Goldschmidt N. CNS prophylaxis in diffuse large B-cell lymphoma: if, when, how and for whom? Blood Rev. 2012;26 (3):97-106. doi:10.1016/j.blre.2011.12.001

24. Graber JJ, Omuro A. Primary central nervous system lymphoma: is there still a role for radiotherapy? Curr Opin Neurol. 2011;24 (6):633-640. doi:10.1097/WCO.0b013e32834cbdef

25. Rubenstein JL, Gupta NK, Mannis GN, LaMarre AK, Treseler P. How I treat CNS lymphomas. Blood. 2013;122(14):2318-2330. doi:10.1182/blood-2013-06-453084

26. Fischer L, Korfel A, Kiewe P, Neumann M, Jahnke K, Thiel E. Systemic high- dose methotrexate plus ifosfamide is highly effective for central nervous system (CNS) involvement of lymphoma. Ann Hematol. 2009;88(2):133-139. doi:10.2214/AJR.07.3919
27. Ferreri AJ, Reni M, Foppoli M, et al. High-dose cytarabine plus high-dose methotrexate versus high-dose methotrexate alone in patients with primary CNS lymphoma: a randomised phase 2 trial. Lancet. 2009;374(9700):1512-1520. doi:10.1016/S0140-6736(09)61416-1

28. Bokstein F, Lossos A, Lossos IS, Siegal T. Central nervous system relapse of systemic non-Hodgkin's lymphoma: results of treatment based on high-dose methotrexate combination chemotherapy. Leuk Lymphoma. 2002;43(3):587-593. doi:10.1080/10428190290012092

29. Wong ET, Tishler R, Barron L, Wu JK. Immunochemotherapy with rituximab and temozolomide for central nervous system lymphomas. Cancer. 2004;101(1):139-145. doi:10.1002/cncr.20339

30. Peñalver F-J, Sancho J-M, de la Fuente A, et al. Guidelines for diagnosis, prevention and management of central nervous system involvement in diffuse large B-cell lymphoma patients by the Spanish Lymphoma Group (GELTAMO). Haematologica. 2017;102 (2):235-245. doi:10.3324/haematol.2016.149120

\section{Publish your work in this journal}

Cancer Management and Research is an international, peer-reviewed open access journal focusing on cancer research and the optimal use of preventative and integrated treatment interventions to achieve improved outcomes, enhanced survival and quality of life for the cancer patient.
The manuscript management system is completely online and includes a very quick and fair peer-review system, which is all easy to use. Visit http://www.dovepress.com/testimonials.php to read real quotes from published authors. 